See discussions, stats, and author profiles for this publication at: https://www.researchgate.net/publication/282913657

\title{
Assessment of driver behavior based on Machine learning approaches in a social gaming scenario
}

Conference Paper · May 2015

CITATIONS

0

6 authors, including:

Gautam Dange

Università degli Studi di Genova

8 PUBLICATIONS 9 CITATIONS

SEE PROFILE

Riccardo Berta

Università degli Studi di Genova

132 PUBLICATIONS 1,892 CITATIONS

SEE PROFILE

Some of the authors of this publication are also working on these related projects:

Project STEM Gaming View project

Project FABRIC View project
READS

680

Pratheep Kumar Paranthaman

Northeastern University

11 PUBLICATIONS 14 CITATIONS

SEE PROFILE

Alessandro De Gloria

Università degli Studi di Genova 212 PUBLICATIONS 2,317 CITATIONS

SEE PROFILE 


\title{
Assessment of driver behavior based on Machine learning approaches in a social gaming scenario
}

\author{
Gautam R.Dange ${ }^{1}$, Pratheep K.Paranthaman ${ }^{1}$, Francesco Belloti ${ }^{1}$, Marco \\ Samaritani ${ }^{1}$, Riccardo Berta ${ }^{1}$, Alessandro De Gloria ${ }^{1}$ \\ ${ }^{1}$ DITEN, University of Genoa, Via Opera Pia 11A, 16145 Genoa, Italy \\ \{ gautam.dange,pratheep.paranthaman, franz, Marco.Samaritani,riccardo.berta, \\ adg?@elios.unige.it
}

\begin{abstract}
The estimation of user performance analytics in the area of car driver performance was carried out in this paper. The main focus relies on the descriptive analysis with our approaches emphasizing on educational serious games, in order to improvise the driver's behavior (specifically green driving) in a pleasant and challenging way. We also propose a general Internet of the Things (IoT) social gaming platform (SGP) concept that could be adaptable and deployable to any kind of application domain. The social gaming scenario in this application enables the users to compete with peers based on their physical location. The efficient drivers will be awarded with virtual coins and gained virtual coins can be used in real world applications (such as purchasing travel tickets, reservation of parking lots, etc.). This research work is part of TEAM project co-funded within the EU FP7 ICT research program.
\end{abstract}

\section{Introduction}

The driver behavior has the direct impact on all the factors that are concerned to efficient driving characteristics such as the road safety, fuel efficacy or the reasonable driving patterns. Also let it be the fatalities or vehicle collisions, it is again a genesis from the behavior of the driver [9] [10]. As the driver behavior holds a significant role in modeling the safe and green driving patterns, there are many models that are emerging under this roof. Providing necessary coaching to drivers under simulated environments would act as a test bed to facilitate the process of designing various safe driving methodologies [11]. Other approaches would be the prior and post analysis of driver behavior with reference to the impact [12] [13], by predicting the outcomes and adjusting the current behavior. However there is a mild patch between the simulation and real-world entity, most of the simulated results have to be adjusted to cope with the real-world situations. These safety and efficient driving measures must be approached from the perspective of system design [10], where the modeling must accommodate the dynamic environment.

The major aim of our project is to inculcate the better driving practices using driver behavior evaluation and social gaming scenario. This research activity was carried as a part of TEAM (Tomorrow's Elastic Adaptive Mobility) project [14]. 
For the real time evaluation, we have collected the vehicle signals from the test run conducted by Centro Ricerche Fiat (CRF) [15] and the test site was located around the CRF office in Trento, Italy. As a viewpoint of our approach, the competitive gaming scenario would induce a good conduct in driving pattern and the evaluators used would ensure that the proper driving behavior is exhibited. As the evaluators are comprised of four different approaches such as Linear Distances to categorize the harsh and smooth driving patterns, Kohonen Neural Networks to map the eventbased behavior and K-Nearest Neighbor approach to plot the relativity between the vehicle signals and to penalize the harsh behaviors attempted by the drivers. The Dynamic Sliding Window captures the deviation from optimal level and tracks the harsh events. Therefore the evaluators are designed to analyze various driving styles and fit to the dynamic environment.

\section{Related Work}

When designing a driver behavior model there are many aspects that are taken into account such as data acquisition tools equipped with the system, extraction of vehicle signals based on the dynamics and most importantly the evaluation methods (algorithms) to process and analyze the vehicle signals to estimate the performance of the driver. Estimation of driver behavior is always a topic of interest in vehicle research sector, out of which we had handpicked certain approaches from state-of-art related to our work and they are as follows.

To estimate the performance of the driver, [1] conducted an experiment involving in acquiring the time-series steering angle data during the lane changes by the driver. The experimental setup was under a controlled simulated environment and driver behavior model was designed using conditional Gaussian model on Bayesian network and there was also a comparison along with traditional Hidden Markov Model. The final reports stated that, there were some minor problems in predicting the sudden changes in the environment, such as drastic change of steering wheel for dynamic happenings. Another comparison approach performed by [2] involved the estimation of driver behavior model by implementing Gaussian mixture model and piecewise auto regressive exogenous (PWARX). The major task was the carfollowing approach, where both of the probabilistic models outperformed each other on various occasions, for instance PWARX produced a good prediction in handling the Gas and brake pedal signals compared to Gaussian mixture model. However both of the algorithms performed well for the limited signals, as in the main focus was on gas and brake pedals only.

The different approach that was implemented in car following task using Dirichlet Process Mixture [3]. The outcome stressed on the fact that, the metrics observed from this experiment can be a good step for future analysis in developing contextadaptive system. These are some of the significant probabilistic models on current practice and let's flip the side of the approach and look out the implementation of driver behavior models using machine learning and adaptive algorithms. The ultimate challenge comes only when things are tested on the real-world applications in a rapidly changing environment, on such aspect the experiment [4] carried out by research team , comprised of "UYANIK", a passenger car equipped with multiple 
sensors and CAN architecture for data acquisition was put up on real-world testing in estimation of driver behavior. This was also a collaborative research conducted by NEDO (Japan), Drive-safe Consortium (Turkey) and the Trans-European Motorway (TEM), Istanbul. Almost the passenger car was equipped with all possible metrics to gather the signal, so as to make the prediction in an enhanced way. Post the data acquisition by CAN-bus, the signals were sent for processing to a SVM (Support vector machines) model, the labelled data were classified by SVM and the results of SVM were forwarded to a Hidden Markov Model (HMM), which performed the final prediction of driver behavior. As the processing involved ample number of input signals, the classification and prediction using the machine learning approach was feasible by the system and the outcomes were formulated in an efficient manner. ANN was used as a major tool to classify the driving styles in various roads in the work carried out by [5]. The vehicle signals such as speed, engine RPM and acceleration were extracted and sent to the remote data center, over there the analysis of data is performed by the neural networks. The ANN would categorize the types of roads (urban, suburban and highway) in which the vehicle was navigating and also the characteristics of the driver as well. However there exists various neural networks architectures, but only Multi-Layer Perceptron (MLP) is preferred predominantly [6]. Different architectures of neural networks must be experimented, so as to emerge out with better solutions for the problems in analysis tasks. On the basis of the state-of-art study on various driver behavior modelling algorithms, there are many evident facts in handling the approach, firstly the probabilistic and mathematical models were considerably well on simulated and controlled driving environments and they exhibited a certain amount of error ratios and uncertainty when it came to real-world applications[1], whereas on other side the Machine Learning algorithms had the tendency to adapt to sudden changes in the environment and performed well with viable accuracy on all aspects such as classification, estimation and importantly the prediction. Another significant issue that has to be noted with respect to adaptive algorithms is, the incoming data signals should be more in number, because that particular aspect makes the task of evaluation a better one. It is also necessary for the evaluation parameters to hold the ability to adapt to environments, as most of the implementation happens on realworld applications. So it's always necessary to design the system architecture with reliable model that would facilitate for the varying environments.

\section{System Architecture}

The major tasks of this approach involves the estimation of driver behavior and to establish a social gaming platform (SGP) to inculcate green driving patterns. The social gaming platform (SGP) comprises of a distributed platform which has three layers: the field devices, the cloud servers and the user personal devices, as displayed in Fig 1. 


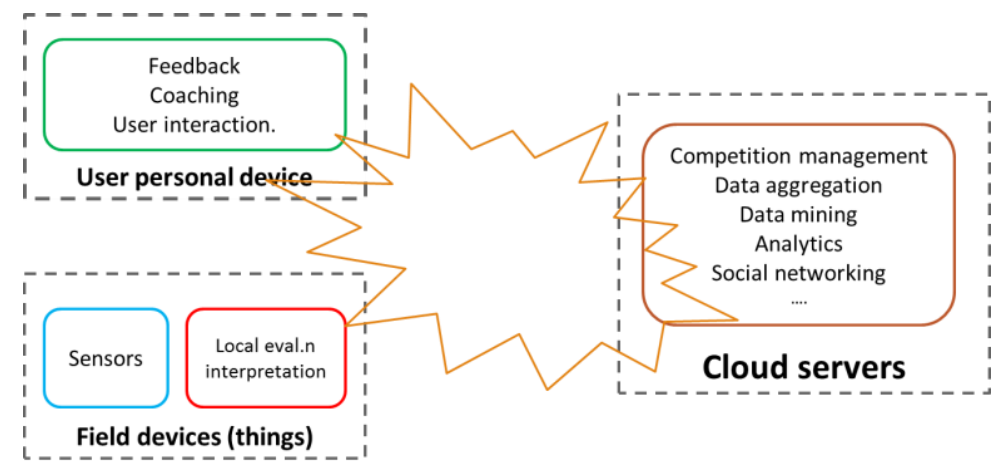

Fig. 1. The IoT Social Gaming Platform Concept

Secondly, for evaluating the driver behavior, the vehicle comprises of an OSGi environment [7], where the evaluators and signal receivers are deployed in the OSGi environment as bundles. Vehicle Signals are transmitted over to VDP consumer bundle using VDP APIs in the OSGi framework and from which the signals are assessed by driver performance assessment module (comprises of the four evaluators) and then the evaluation scores are sent to the cloud servers namely Virtual coins server and competition server and they are updated constantly. The data from the respective servers (virtual coins and competition server) can be accessed using a smartphone application called SG-CB (Serious games and community building).

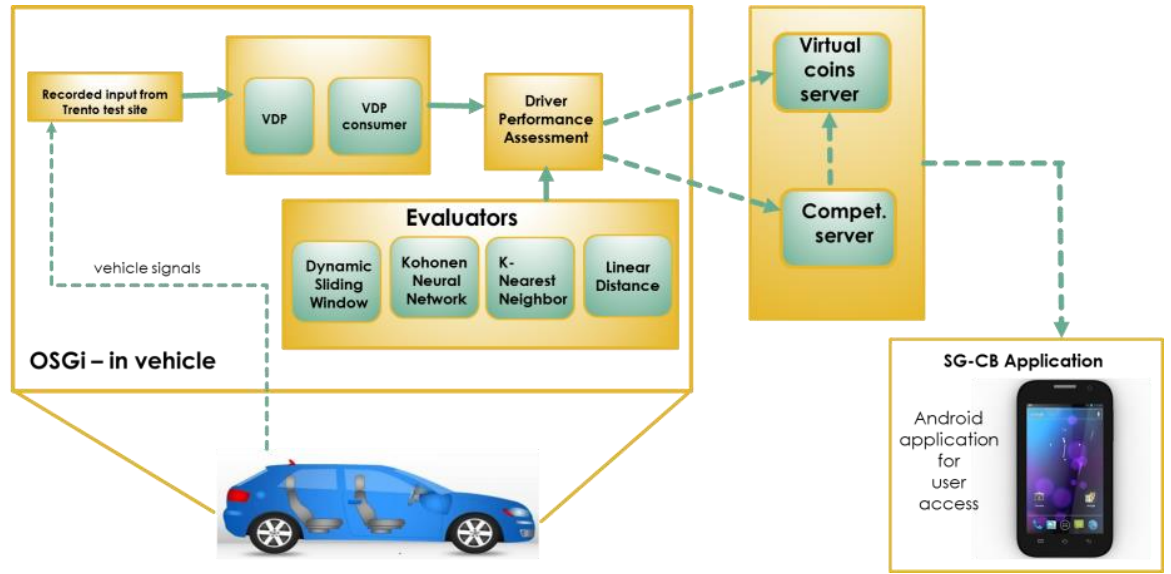

Fig. 2. System overview comprising the entire control flow with the recorded vehicle signals from the test run conducted at Trento, Italy.

In the OSGi environment, the signal values are interpreted from the recorded data and it is transferred to VDP Consumer using VDP APIs. VDP Consumer is another 
bundle running in OSGi environment, which receives data from Trento bundle and subsequently sends the signals to different evaluator applications, which are running in OSGi environment and have subscribed for signals. Due to this approach of pipelining, the addition of new evaluator would not require any code changes, and modification of the configuration file would do this job.

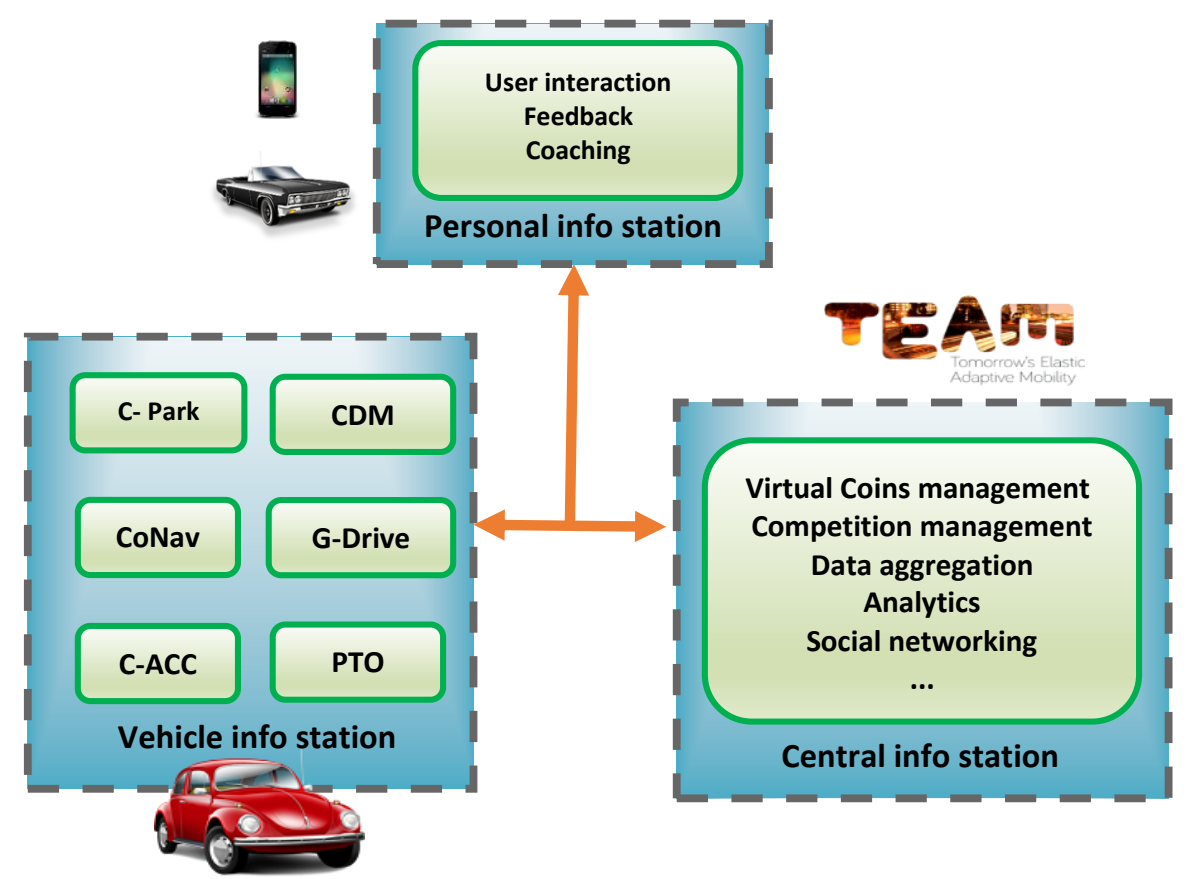

Fig. 3. Application in the Automotive Domain

The internet of the things (IoT) social gaming platform (SGP) in TEAM implementation on automotive domain is illustrated in Fig.3. Over here the different applications exploiting vehicular and other mobility information contributes to the player performance evaluation, thus enabling the game dynamics.

\section{Test Site description}

The test run was conducted by Centro Ricerche Fiat (CRF) and the test site was located around the CRF office in Trento, Italy and the test site comprised of various road segments such as urban, suburban and the mixture of both. The test site was divided into four major zones such as Tangenziale, Viale Verona, Misto and Via 
Nationale and expands around 13 Kilometers, with approximate completion time of the entire run around 28-30 Minutes.

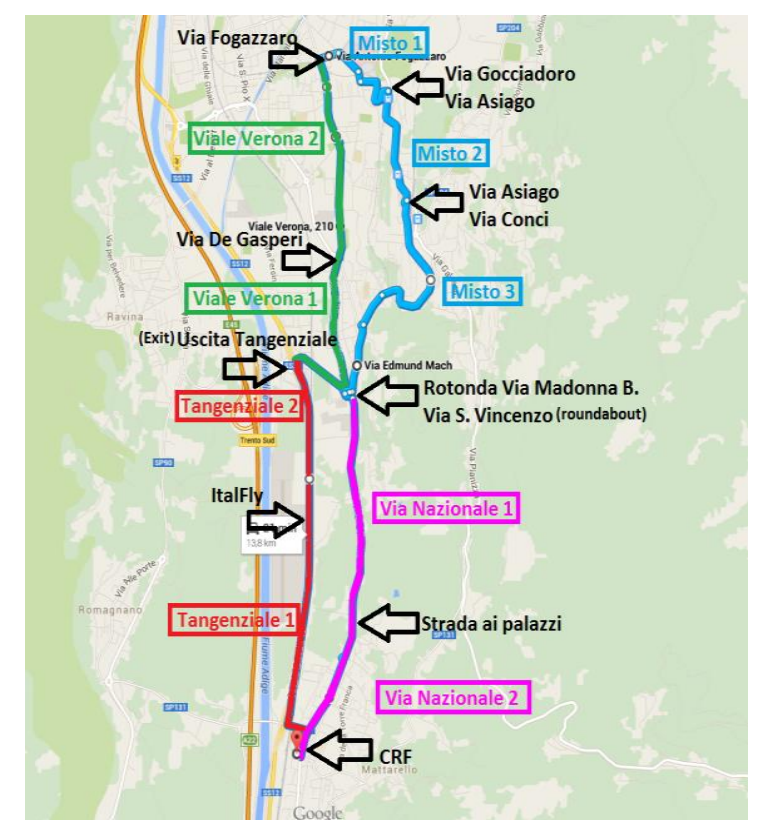

Fig. 4. Map of the test site in Trento, Italy with locations comprising of urban and sub urban zones. The red route highlighted on the map is the suburban zone (Tangenziale) and the green route is of urban zone (Viale Verona). The blue and purple routes are the mixture of urban and suburban zones.

\section{Driver Performance Assessment}

The four evaluators used are:

- Kohonen Neural Networks

- K-Nearest Neighbors

- Linear Distances

- Dynamic Sliding Window

While designing the evaluators for our process, we considered certain metrics to estimate the behavior of driver such as capturing harsh events, mapping the relativity of signals and segmenting the signal patterns to plot the coarse driving behavior. By having all these considerations on ground, we developed four evaluators to handle the metrics stated. When it comes to the issue of processing the large amount of data and capturing the patterns from the given data, the data 
mining algorithms will be the best tool to handle the circumstance with at most robustness. As our process involves the classification of the raw signals into harsh and smooth patterns based on events, we preferred Kohonen Neural Networks [8], which is a classifier based on unsupervised learning approach and it has the ability to classify the data into clusters without any reference samples. The dynamic sliding window was specifically used to track the harshness in the signal patterns that are ranging continuously without any expeditious changes (such as vehicle speed signal pattern, in which the rise and fall happens gradually). The Linear Distances approach was used to segment the signals based on a thresholding point .Another evaluation metric involves the mapping of relativity between signals and we used K-Nearest Neighbors algorithm to handle the task, here we plotted the harshness by analyzing the conflict between the related signals (Example: Speed vs. Brake).

\subsection{Kohonen Neural Networks}

The predominant architectures of Neural Networks work on supervised learning approach, but as our classification is not dependent on samples and also the pattern of input signals (Ex: acceleration, RPM and speed) is not constant and varies depending on the drivers, so we preferred an unsupervised learning architecture for neural networks (i.e.) Kohonen Neural Networks. The network architecture consists of two input neurons and two output neurons with the connection weights. The acceleration and brake signals are broken into two samples and sent to the neural networks. Based on the input patterns only one output neuron is fired and it is considered as the winning neuron, on subsequent iterations the network forms the clusters in the attained input patterns. As a consolidation the output clusters consists of harsh and smooth patterns and then the data is further processed and the harsh patterns based on the events are penalized.

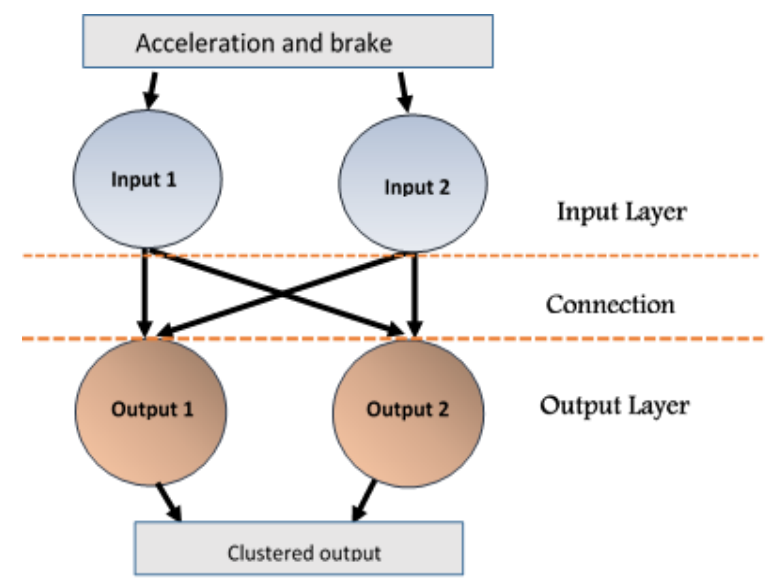

Fig.5. Kohonen Neural Networks structure used for evaluation. The neural networks structure represents the parameters used such as the input and output neurons with the signals that were evaluated. 
Once the harshness is calculated the virtual coins are awarded for the users on the overall performance and the updates of virtual coins are forwarded to the virtual coins server. In the time slots allotted for competitions, the scores for the performance of the user for respective competitions are forwarded to the competition server. The same architecture of neural networks was used for the evaluation of acceleration and brake signals separately in order to maintain the bifurcation of the clusters. The average score of the two independent evaluations are calculated and it is comprised under the application name of Green drive 2. Post the calculations, the scores are transmitted to the server.

\subsection{K-NN (Nearest Neighbors)}

The significant reason for using K-NN is to map the relativity between the speed and brake signals and to penalize when the conflict between them increases (Example: when the vehicle is at high speed, the brake should be applied minimally, If the driver applies high pressure brake, then the action will be highly penalized). The samples of harsh patterns are supplied as a training set to the algorithm and from which the specific harsh values are picked up and penalized. The signals such as speed and brake values are supplied as inputs to the algorithm and the harsh patterns of these signals are provided as a training set to the algorithm. In the K-NN Implementation here, $K=1$, that is nearest neighbors reference used to calculate the penalty for the single signal value. For each signal value, the algorithm refers to the sample set to calculate penalty value. We have control on penalty and award values, and they can be set as per the changes in the criteria. This gives the possibility of customization to change expected conditions and deviations. K-Nearest Neighbors approach can evaluate the relativity of more than one signal at the same time, such as Steering wheel vs. Speed and Steering wheel vs. brake.

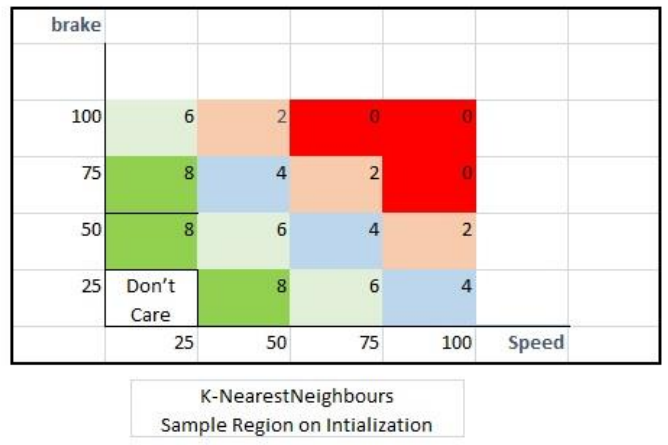

Color Definitions

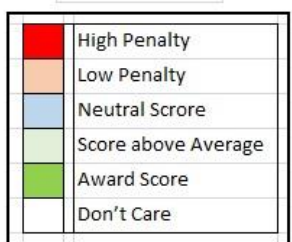

Fig. 6. Representation of the evaluation pattern of K-NN algorithm having multiple regions with different colors. The algorithm evaluates and maps the relativity between speed and harsh brake pattern. In case of high speed, if user applies harsh break, it will result in high penalty, which in turn will reduce the score. 


\subsection{Linear Distances}

Unlike Kohonen Neural Networks, where the batch processing is performed, the Linear Distances holds a thresholding based evaluation in which every single signal is processed and evaluated for harshness. The metrics for evaluating harshness is devised using the linear equation from which the certain criterion forming the boundaries for penalty and award regions are derived.

$$
\mathrm{y}=\mathrm{mx}+\mathrm{b} .
$$

The slope (m) and intercept (b) for the linear equation are supplied through the $\mathrm{XML}$ file and from which the penalty values are calculated.

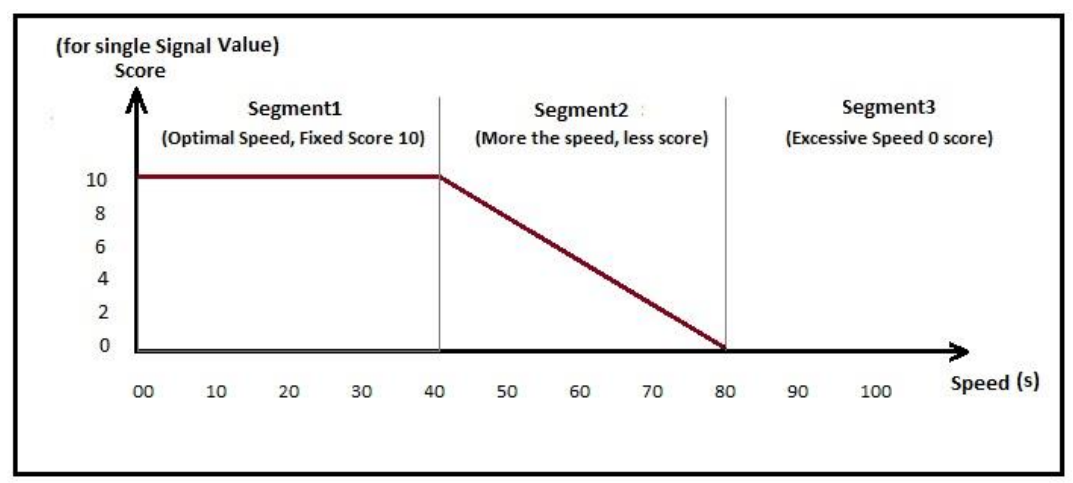

Fig. 7. Evaluation criterion for Linear Distances with representation of segments involving variation of speed signal values.

The above figure represents the processing of speed signal using Linear Distances approach, the entire space is divided into three segments (segment 1, segment 2 and segment 3). Each segment holds the set of limits such as segment 1 has the limit from 0 to 40 and segment 2 holds the limit from 41 to 80 and the values exceeding 80 will be gathered in segment 3 . Let's assume that the vehicle speed is around $35 \mathrm{Km} / \mathrm{h}$, now this signal would fall in segment 1 where the limit ranges from 0 to 40 and it is considered to be optimal driving and the user would get the score of 10 . In another case let the vehicle speed be $60 \mathrm{~km} / \mathrm{h}$, this signal would fall under segment 2, where the limit ranges from 41 to 80 and as the speed limit exceeds the optimal criteria the score would be 5 . Thus, when the speed exceeds certain limits the scores would reduce considerably. The signals such as acceleration, RPM and speed are evaluated using Linear Distances in our approach, the results of the evaluation will be sent to the virtual coins and competition server. 


\subsection{Dynamic Sliding Window}

Dynamic Sliding Window is an event-based signal evaluation. The algorithm comprises of two states (i.e.) standard window and penalty window. In this implementation, the Dynamic Sliding Window is evaluating every single Signal. When the signals are in optimal range, state is considered to be standard (normal region for signals) and when signal values deviate above from the optimal level, then the state is considered to enter the penalty window. Once the signal starts to deviate above the optimal level, the penalty window starts and remains till the point when it comes back to optimal level. The size of penalty window and the deviation amount in that period of time are the factors which are getting evaluated under this algorithm. For example, if the user exceeds a particular speed limit, then the state changes from normal window to Penalty window. If speed limit is $60 \mathrm{~km} / \mathrm{h}$, then user who drivers at speed of $65 \mathrm{~km} / \mathrm{h}$ for 1 minute will deserve same penalty as of another user, who is driving at the speed of $90 \mathrm{~km} / \mathrm{h}$ for 10 seconds.

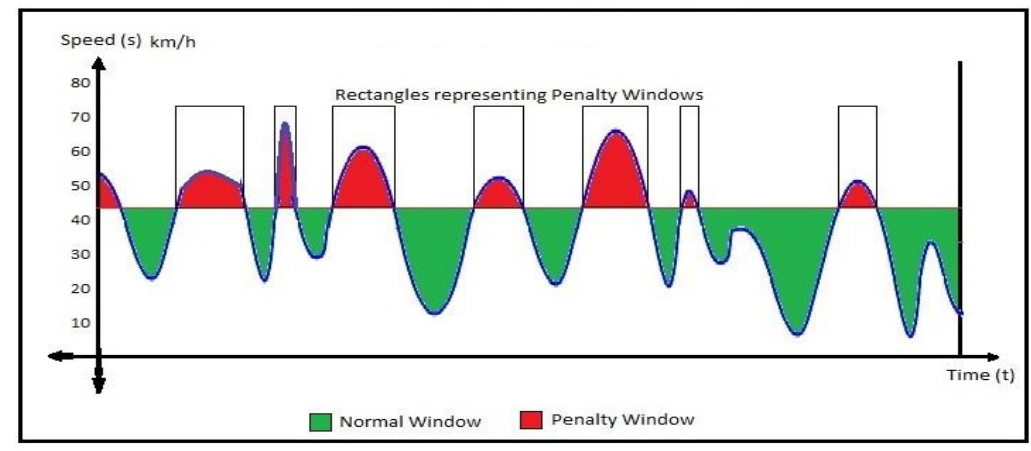

Fig. 8. Representation of Dynamic sliding window with the evaluation of speed signal

\section{Social Gaming prototype scenario}

The social gaming scenario consists of various competitions (drive around a location, for example: a city) comprising a self and social comparisons, where the competition involves the participation of multiple users and each user in the competition will be evaluated and granted a score out of 100 (scores will be calculated based on the results of the evaluators used). The competition user holding a highest score will be declared as the winner of competition and will be awarded with the virtual coins. The users can get virtual coins at any time by exhibiting safe and green driving behavior, as virtual coins are continuous evaluation of driver performance independent of peers, routes and competitions. In order to manage the signals evaluated by the evaluators and to make the scoring pattern tractable, we labelled each evaluator with an application name, where each application would constitute a set of signals for the evaluation and transmit the evaluation results under the roof of an application name 
Table 1. The List of evaluators, signals evaluated, score contribution and the application names associated with the evaluators are displayed in the table.

\begin{tabular}{cccc}
\hline $\begin{array}{c}\text { Application } \\
\text { Name }\end{array}$ & Evaluator & Signals Evaluated & $\begin{array}{c}\text { Score } \\
\text { Contribution }\end{array}$ \\
\hline Green drive 1 & Linear Distances & Acceleration and RPM & $20 \%$ \\
Fluid traffic & Linear Distances & Speed and Fuel Consumption & $20 \%$ \\
Green drive 2 & $\begin{array}{c}\text { Kohonen Neural } \\
\text { Networks }\end{array}$ & Acceleration and brake & $20 \%$ \\
Green drive 3 & $\begin{array}{c}\text { K-Nearest } \\
\text { Neighbors }\end{array}$ & Speed and brake & $20 \%$ \\
Green drive 4 & $\begin{array}{c}\text { Dynamic Sliding } \\
\text { Window }\end{array}$ & Speed & $20 \%$ \\
\hline
\end{tabular}

\section{Results}

In order to compare the efficacy of the four evaluators, we performed the test in evaluating the acceleration signal with all the four evaluators on the time interval of 2 minutes. The below figure represents the evaluators test results (four evaluators) of acceleration signal for 8 minutes.

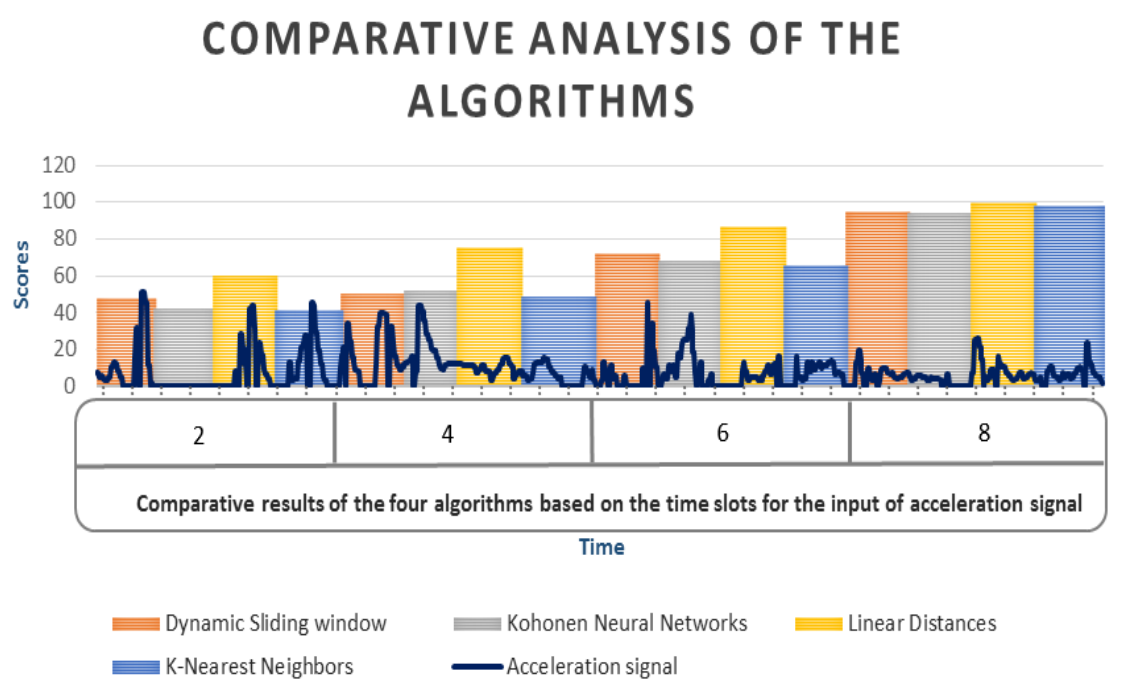

Fig. 9. Comparative analysis of the algorithms 
From the observations of the four evaluators, it can be noted that the evaluators have different grading patterns, where the Kohonen Neural Networks have a penalizing feature for minor harsh patterns as well and this approach can be used for correcting the mistakes in novice driving style. Whereas, the K-NN has a nominal penalizing criterion in which certain harsh patterns are provided as a sample set to the system and specific harsh patterns are captured and penalized. The Linear Distances has a penalizing scheme based on segments with standard flow of virtual coins and dynamic sliding window's evaluation has an event based penalizing criterion. These four evaluation approaches have been designed to provide a viable solution to the various needs of the user and most importantly for the betterment of the driver behavior. The dimensions involved in evaluation mechanisms can facilitate the process of model adaptation, because model adaptation is one common problem in evaluation methods used for tracking the driver behavior.

\section{Conclusion}

As a consolidation, we have implemented set of algorithms including the significant Machine Learning approaches to evaluate the driver behavior based on various vehicle signals acquired from the test run conducted by Centro Ricerche Fiat (CRF) in Trento, Italy. By using certain strategies in designing the evaluation metrics such as event-based, relativity mapping of signals and segmenting, we have attained a viable solution to track the driver behavior on all occasions and driving standards. This methodology would be a stable approach as it is a collection of model adaptive tools and the tests involved were carried out on data acquired from real-world scenario. The model adaptive approach has the tendency to accommodate the needs of the user and also can be resilient for dynamic environments. The system can be further extended in future by integrating different applications (Public transport Optimization, Collaborative navigation, collaborative driving and maneuvering) and a comparison approach can be introduced, with which the users can gauge their performance and compare with their peers. Finally, the social gaming chain introduced in this approach would induce a competitive learning traits and enhances the efficiency of driving. Henceforth, this approach has coherent mechanism to inculcate safe and green driving patterns and caters the various types of drivers with the aid to improvise the driving proficiency. 


\section{References}

1. S. Tezuka, H. Soma, and K. Tanifuji, “A Study of Driver Behavior Inference Model at Time of Lane Change using Bayesian Networks," pp. 2308-2313, 2006.

2. P. Angkititrakul, C. Miyajima, and K. Takeda, "Modeling and Adaptation of Stochastic Driver-Behavior Model with Application to Car Following," no. Iv, pp. 814-819, 2011.

3. P. Angkititrakul, C. Miyajima, and K. Takeda, "Impact of Driving Context on Stochastic Driver-Behavior Model : Quantitative Analysis of Car Following Task," 2012.

4. H. Erdog, A. Ozgu, B. Akan, H. Karabalkan, V. Sezer, M. Karaca, M. Abbak, and K. Eritmen, "In-Vehicle Corpus and Signal Processing for Driver Behavior," pp. 23-44, 2009.

5. J. E. Meseguer, C. T. Calafate, J. C. Cano, and P. Manzoni, "DrivingStyles: A smartphone application to assess driver behavior," in Proceedings International Symposium on Computers and Communications, 2013.

6. M. Dougherty, "A review of neural networks applied to transport," Transportation Research Part C Emergerging Technologies, vol. 3, no. 4, pp. 247-260, 1995.

7. C. Lee, D. Nordstedt, and S. Helal, "Standards, Tools \& Best Practices Enabling Smart Spaces with OSGi," Pervasive Computing IEEE, vol. 2, no. 3, pp. 89-94, 2003.

8. S.-J. H. S.-J. Huang and C.-C. H. C.-C. Hung, "Genetic algorithms enhanced Kohonen's neural networks," Proceedings of the ICNN'95 - International Conference on Neural Networks, vol. 2, pp. 1-5, 1995.

9. J. C. McCall and M. M. Trivedi, "Driver behavior and situation aware brake assistance for intelligent vehicles," Proceedings of IEEE, vol. 95, no. 2, pp. 374-387, 2007.

10. S. S and G. G. Ray, "Human factors in safe driving-A review of literature on systems perspective, distractions and errors," Proceedings - 2012 IEEE Global Humanitarian Technology Conference, GHTC 2012, pp. 83-88, 2012.

11. V. Punzo and B. Ciuffo, "Integration of driving and traffic simulation: Issues and first solutions," IEEE Transactions on Intelligent Transportation Systems, vol. 12, no. 2, pp. 354-363, 2011. 
12. N. M. Nor, A. Wahab, N. Kamaruddin, and H. Majid, "Post accident analysis of driver affection," Proceedings of International Symposium on Consumer Electronics ISCE, pp. 278-283, 2011.

13. J. Gong, "Driver pre-accident behavior pattern recognition based on dynamic radial basis function neural network," Proceedings 2011 International Conference on Transportation Mechanical and Electrical Engineering (TMEE), pp. 328-331, 2011.

14. "TEAM," [Online]. Available: http://www.collaborative-team.eu/overview/. [Accessed: 13-April-2015].

15. “CRF," [Online]. Available: http://www.crf.it/en-US/Company/Pages/ CompanyProfilo.aspx. [Accessed: 08- May- 2015]. 